\title{
Über die Entscheidbarkeit der formalen Erreichbarkeit von Prozeduren bei Monadischen Programmen
}

\author{
Lippe, Wolfram-Manfred
}

First published in:

Programmiersprachen, S. 124 - 134, Berlin 1976, ISBN 3-540-07619-0

Münstersches Informations- und Archivsystem multimedialer Inhalte (MIAMI)

URN: urn:nbn:de:hbz:6-69329426436 
ÜBER DIE ENTSCHEIDBARKEIT DER FORMALEN ERREICHBARKEIT

VON PROZEDUREN BEI MONADISCHEN PROGRAMMEN

\author{
Lolfram-Manfred Lippe \\ Institut für Informatik und angewandte Mathematik \\ Christian-Albrechts-Universität, D-23 Kiel
}

\title{
1. Einleitung
}

Beim Bau van Übersetzern für ALGuL-ähnliche Programmiersprachen erfurdert die Behandlung von Prozeduren einen besonderen Aufwand. Deswegen ist ea wünschenswert, Algorithmen zu besitzen, die gewisse Eigenschaften van Programmen mit Prazeduren bereits zur libersetzungszeit entscheiden können, um so durch eine differenziertere Behandlung der Prozeduren unnötigen Aufwand einzusparen und effizientere Zielprogramme zu erzeugen. Zu diesen Eigenschaften gehören z.B.

1) die formal karrekte Parameterübergabe in einem Programm mit Prozeduren,

2) die formale Erreichbarkeit einer Prazedur,

3) die formale Rekursivität einer Prozedur,

4) die formal starke Rekursivität einer Prazedur,

5) die Makro-Programm-Eigenschaft eines Programms mit Prozeduren.

Bei Programmen mit formal korrekter Parameterübergabe braucht zur Laufzeit nicht geprüft zu werden, ob aktuelle und formale Parameter zueinander passen. Nicht formal stark rekursive Prozeduren und erst recht nicht formal rekursive Prozeduren dürfen wie Blöcke behandelt werden, d.h. ihre Festspeicher kännen innerhalb der Festspeicher der kleinsten umfassenden Prozeduren untergebracht werden, und eigene Display- (Index-) Register sind unnätig. Programme mit der Makro-Programm-Eigenschaft benötigen zur Laufzeit überhaupt keine Prazedurarganisation, was die Laufzeit erheblich verkürzt. Gewiß kann die makroartige Expansion des Originalprogramms zu unerträglichen statischen Programmlängen führen, in der Systemprogrammierung, insbesandere beim Compilerbau, wird man den erhähten Speicherbedarf tratzdem in Kauf nehmen, weil sonst wegen der häufigen Prozeduraufrufe ein relativ großer Zeitverlust eintritt.

Für ALGQL 60 sind die Eigenschaften 1)-5) unentgcheidbar, ebenso 2)-5) für ALGOL 68, selbst wenn nur Identifikatoren als aktuelle Parameter zugelassen sind $[5,6]$.

Die bls jetzt bekannten Resultate bzgl. der formalen Erreichbarkeit von Prozeduren in generellen Programmen lassen sich durch folgendes Diagramm reprä- 
sentieren, das die Programme nach maximaler Parameterzahl P pro Prozedur und nach maxi.naler Prozedurschachtelungstiefe $S$ einteilt:

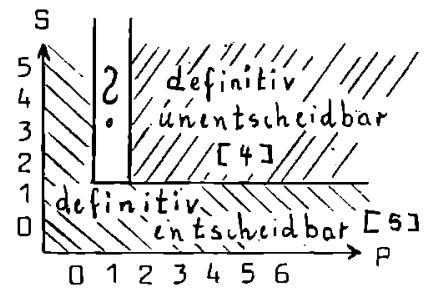

Wir wollen jetzt eine umfangreiche Programmteilklasse definieren, und für sie die Entscheidbarkeit nachzuweisen versuchen. Der definitive Entscheidbarkeitsbereich sollte natürlich möglichst weit über den obigen hinausragen.

Zum Beweis der Unentscheidbarkeit der formalen Erreichbarkeit von Prozeduren wird in [5] für jedes Post'sche Korrespandenzsystem $\mathscr{L}$ ein Programm $\pi_{2}$ mit einer Prozedur M1 effektiv konstruiert, so daß $\mathscr{W}$ genau dann eine Lösung hat, wenn $M 1$ in $\pi_{2}$ formal erreichbar ist. Es fält auf, daß in $\pi_{2}$ die entscheidenden Prazeduranweisungen $a_{0}\left(a_{1}, \ldots, a_{n}\right)$ von der Art sind, dab alle formalen Identifikatoren $a_{i}$ paarweise verschieden sind. Wenn wir nun ein Programm monadisch nennen, falls in jeder Prozeduranweisung $a_{0}\left(a_{1}, \ldots, a_{n}\right)$ für die formalen Identifikatoren $a_{i}, a_{j} a_{i}=a_{j}$ ist, so führt der zitierte Beweis zu der Vermutung, daB die formale Erreichbarkeit von Prozeduren für monadische ALGOL 60-Programme entscheidbar ist.

Oiese Vermutung kann noch nicht vallständig bewiesen werden. Der Beweis ist jedoch für zwei graße Teilklassen monadischer Programme müglich, erstens für monadische Programme mit maximaler Prozedurschachtelungstiefe 2 und maximaler Parameterzah1 2 pro Prozedur, zweitens für monadische Programme mit maximaler Prozedurschachtelungstiefe 3 und maximaler Parameterzahl 1 pro Prozedur. Aus dem Beweis des ersten Falls ist ersichtlich, daß die Beschränkung der Parameterzahl im Grunde unerheblich ist. Im ersten Fall benutzen wir als Hilfsmittel Baumsysteme [B], im zueiten Fall Stacksysteme [1]. Von beiden Systemen ist bekannt, daß das Zustandserreichbarkeitsproblem algarithmisch lösbar ist. Der Einfachheit halber beschränken wir uns auf die Sprache ALGLL 60-P (P=pure), die gegenüber ALGOL 60 falgende hauptsächlichen Einschränkungen und Veränderungen besitzt:

a) keine Funktionsprozeduren,

b) kein "call by value",

c) nur Identifikatoren als aktuelle Parameter von Prozeduranweisungen,

d) alle Prozedurrümpfe sind in Rumpfklammern eingeschlossen,

e) leerer Spezifikatiansteil im Prozedurkopf.

Für eine vollständige Definition von ALGOL 60-P siehe [5]. 


\section{Definitionen}

Ein formales Algol 60-P-Programm $\pi$ ist eine Zeichenkette aus Grundsymbolen, die durch die formalen fegeln der kontextfreien Grammatik of für ALGOL 60-P auf das Axiom 〈program〉 reduziert werden kann und für die die falgende Eigenschaft gilt: Zu jedem auftretenden Identifikatar gibt es genau ein zugehöriges definierendes Auftreten. Ein formales Programm heißt übersetzbar, falls jedes Auftreten einer Kanstanten oder eines Identifikators gemäß seiner Definition vernünftig erfalgt. Zwei formale Programme heißen identisch, falls sie sich nur durch eine zulässige Umbenennung van Identifikatoren unterscheiden, und wir wollen in Zukunft nicht zwischen identischen Programmen unterscheiden. Ein farmales Programm heißt ausgezeichnet, falls verschiedene definierende Varkommen von Identifikatoren durch verschiedene Identifikatoren bezeichnet sind. Ein farmales Programm heißt partiell übersetzbar, falls nach dem Ersetzen aller Prozedurrümpfe durch leere Rümpfe das sich so ergebende Programm übersetzbar ist.

Definition: Sei $\pi$ partiell übersetzbar. Ein Programm $\pi^{\prime}$ heiBt Erqebnis aug $\pi$ durch Anwendung der Kopierregel ( $\pi$ - $\pi^{\prime}$ ) falls gilt:

Sei $f\left(a_{1}, \ldots, a_{n}\right)$ eine Prozeduranueisung im Hauptprogramm von $\pi$. Sei ferner groc $f\left(x_{1}, \ldots, x_{n}\right) ;\{\sigma\}$ die zugehörige Prozedurdeklaration. Die partielle übersetzbarkeit von $\pi$ garantiert, daß die Anzahl der aktuellen und formalen Parameter übereinstimmt. Sei ferner $\pi$ o.B.d.A. ausgezeichnet. Dann wird $f\left(a_{1}, \ldots, a_{n}\right)$ durch einen modifizierten Rumpf $\left\{\sigma^{\prime}\right\}$ ersetzt, indem die formalen Parameter $x_{i}$, die in $\{\sigma\}$ auftreten, durch die entsprechenden aktuellen Parameter $a_{i}$ ersetzt werden.

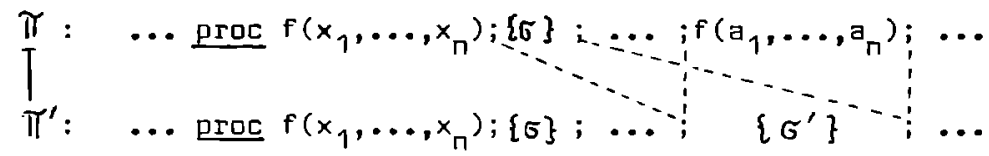

Durch Umbenennung aller Identifikatoren in $\sigma^{\prime}$, die in $\sigma^{\prime}$ selbst gebunden sind (lokal), wird $\pi^{\prime}$ ausgezeichnet. $\left\{\sigma^{\prime}\right\}$ heißt erzeugter Block; die klammern \{\} bei $\left\{\sigma^{\prime}\right\}$ heiBen Aufrufklammern im Gegensatz zu den Rumpfklammern \{\} bei $\{\sigma\}$.

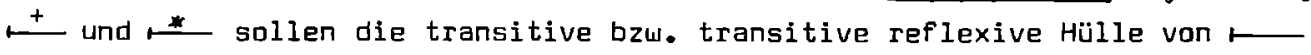
bezeichnen. Ein formales Programm $\pi$ heiBt original, falls \{\} nur als Rumpfklammern auftreten.

Definition: Sei $\pi$ ariginal. Die Menge $T \pi$ mit

$$
\begin{aligned}
& \mathrm{T}_{\pi}:=\left\{\pi^{\prime} / \pi^{*}+\pi^{\prime}, \pi^{\prime}\right. \text { besitzt höchstens einen innersten } \\
& \text { erzeugten Block\} }
\end{aligned}
$$

heißt Ausführungsbaum von $\pi$.

Mit IGB $\left(\pi^{\prime}\right)$ bezeichnen wir den innersten erzeugten Block von $\pi^{\prime}$. Entfernen wir bei einem Programm $\pi^{\prime} \epsilon T \pi$ alle Prozedurdeklarationen, so erhalten wir das zugehärige Hauptprogramm $\pi_{m}^{\prime}$. Ersetzen wir alle Prazeduranweisungen in $\pi_{m}^{\prime}$ durch ein Sonderzeichen, z.B. call, so erhalten wir das reduzierte Hauptprogramm $\mathbb{T}_{I}$ 
$\operatorname{von} \pi$.

Definition: $T_{\Gamma \pi}:=\left\{\pi_{\Gamma}^{\prime} / \pi^{\prime} \in T_{\pi}\right\}$ heilt reduzierter Ausführungsbaum von $\pi$. Definition: Zwei originale Programme heißen formal äquivalent, falls ihre reduzierten Ausführungsbäume identisch sind.

Definition: Eine Prozedur in einem originalen Programm $\pi$ heiBt formal erreichbar, falls ein Programm $\pi^{\prime} \in T_{\pi}$ ex., so daß IGB $\left(\pi^{\prime}\right)$ ein modifizierter Rumpf einer Kopie dieser Prozedur ist.

$\checkmark$

Im folgenden werden wir uns mit der formalen Erreichbarkeit bei einem besonderen Typ van Programmen, den monadischen Programmen, beschäftigen.

Definition: Ein Programm $\pi$ hei bt monadisch, wenn in jeder Prozeduranueisung $a_{a}\left(a_{1}, \ldots, a_{n}\right)$ für die formalen Identifikatoren $a_{i}, a_{j}$ gilt $a_{i}=a_{j}$.

Um die Beweise zu vereinfachen, machen wir ferner die unerhebliche Einschränkung, daß es neben Prozedurdeklarationen keine weiteren Deklarationen und neben Prozeduranueisungen keine weiteren Anweisungen geben soll.

Zum Beweis, daß die formale Erreichbarkeit von Prozeduren bei monadischen Programmen entschei bbar ist, führen wir unsere ALGOL 60-P-Programme in formal äquivalente Programme der modularen Programmiersprache ALGaL 60-P-G (closureSprache) über. Prozedurdeklarationen in ALGOL 6D-P-G haben die form

(*) $\quad$ proc $f\left\langle y_{1}, \ldots, y_{m_{f}}\right\rangle\left(x_{1}, \ldots, x_{n_{f}}\right) ;\{s\} ;$

Die Identifikatoren $y_{1}, \ldots, y_{m_{f}}$ heiBen formale Parameter neuer Art, $x_{1}, \ldots, x_{n_{f}}$ sind die formalen Parameter alter Art. Die klammern fallen weg, falls $m_{f}$ bzw. $n_{f}$ Null ist. Alle übrigen Deklarationen sehen wie in ALGOL 60-P aus. Ein Term ist eine endliche Zeichenreihe, die nach folgenden Regeln gebildet wird:

1. Identifikatoren sind Terme.

2. Wenn $\psi$ Identifikator ist und $\tau_{1}, \ldots, \tau_{m}, m \geqslant 1$, Terme sind, dann ist auch $\left.\psi<\tau_{1}, \ldots, \tau_{m}\right\rangle$ ein Term. $\tau_{i}$ heißt aktueller Parameter neuer Art.

Aus einem formalen ALGCL 60-P-Programm $\pi^{\prime}$ entsteht ein formales ALGOL 60-P-GProgramm $\pi$ dadurch, daß die Prozedurköpfe in $\pi^{\prime}$ durch neue Köpfe der Farm (*) ersetzt werden und daß man in $\pi^{\prime}$ angewandt vorkommende Identifikatoren durch Terme auswechselt, so daß jeder Identifikator genau eine Definition besitzt. Auch alle übrigen Begriffe lassen sich ohne Schwierigkeiten auf ALGDL 60-P-G ausweiten.

Der Unterschied zwischen den beiden Sprachen liegt in der Methode, wie man Prozeduren $f$, die durch formale Prozeduranweisungen aufgerufen werden, durch aktuelle Parameter versargt. In ALGOL 60-P werden alle aktuellen Parameter im Moment des Aufrufs übergeben. In ALGOL 60-P-G werden die aktuellen Parameter alter Art ebenfalls im Moment des Aufrufs übergeben, während die aktuellen Parameter neuer Art so betrachtet werden kännen, als ob sie bereits vorher übergeben worden wären, als der Prozeduridentifikatar $f$ als aktueller Parameter eines vorange- 
gangenen Aufrufs auftrat. Auf Grund dieser Wirksamkeit der formalen Parameter neuer Art ist es möglich, jedes ariginale ALGoL 60-P-G-Pragramm in ein farmal äquivalentes ALGOL 60-P-G-Programm ahne Prazedurschachtelung (modular) effektiv umzuformen.

3. Baumsysteme

Betrachtet man nur Programme mit maximaler Parameterzahl 1 und maximaler Prozedurschachtelungstiefe 2, so besitzen alle Terme, die bei den Prozeduranweisungen des Ausführungsbaumeg auftreten können, die Form einer linearen Zeichenkette. Dieser Spezialfall wurde bereits in [7] untersucht, und die Entscheidbarkeit der formalen Erreichbarkeit mit Hilfe von regulären kanonischen 5ystemen gezeigt. Erhöht man bei den Programmen die Parameteranzahl, bzw. die Prozedurschachtelungstiefe, so besitzen die einzelnen Terme eine baumartige Struktur. Im Gegensatz zu den Baumautomaten, wie sie von Brainerd, Doner usw. betrachtet wurden, benätigen wir jedoch Systeme, die eine Modifikation des Eingabebaums durch Veränderung der Wurzel erlauben. Deswegen benutzen wir Baumsysteme, wie sie von Rounds [B] eingeführt wurden.

Definition: Ein markiertes Alphabet ist ein Paar $(\Sigma, \Gamma)$, wabei $\Sigma$ eine endliche Menge und $r: \Sigma \longrightarrow \mathbb{N}$ eine Abbildung ist. Sei ferner $\Sigma_{\Pi}$ durch

$$
\Sigma_{n}:=\{\sigma \in \Sigma / \Gamma(\sigma)=n\}
$$

definiert.

Hiermit lassen sich nun $\underline{\Sigma}$ - Terme (日äume) definieren:

Definition: Sei $(\Sigma, r)$ ein markiertes Alphabet. Die Menge $\mathcal{J}_{\Sigma}^{0}$ ist die kleinste Menge, derart dab
(a) $\Sigma_{0} \subsetneq \zeta_{\Sigma}^{0}$
(b) falls $t_{0}, \ldots, t_{n-1} \in \mathcal{J}_{\Sigma}^{0}$ und $\sigma \in \Sigma_{n}>\sigma\left\langle t_{0}, \ldots, t_{n-1}\right\rangle \in \zeta_{\Sigma}^{0}$

Was ein Unterterm eines Terms 1st dürfte klar sein. Die $t_{1}, 0 \leqslant i \leqslant n-1$, in $G\left\langle t_{0}, \ldots, t_{n-1}\right\rangle$ bezeichnen wir auch als unmittelbare Unterterme, $t_{0}$ als unmittelbaren linken Unterterm und $t_{n-1}$ als unmittelbaren rechten Unterterm. Um Terme miteinander vergleichen zu kännen, benötigen wir noch Definition: Zwei $\Sigma$-Terme $G\left\langle t_{a}, \ldots, t_{n-1}\right\rangle$ und $\sigma^{\prime}\left\langle t_{0}^{\prime}, \ldots, t_{k-1}^{\prime}\right\rangle$ heißen fastidentisch, falls $n=k$ und $t_{1}=t_{i}^{\prime}$ fïr $i=0, \ldots, n-1$. Definition: Seien $\sigma\left\langle t_{0}, \ldots, t_{n-1}\right\rangle$ und $\sigma^{\prime}\left\langle t_{0}^{\prime}, \ldots, t_{k-1}^{\prime}\right\rangle$ zwei $\Sigma-T e r m e$. Dann heiBt $\sigma^{\prime}\left\langle t_{0}^{\prime}, \ldots, t_{k-1}^{\prime}\right\rangle$ Fastunterterm von $\sigma\left\langle t_{0}, \ldots, t_{n-1}\right\rangle$, falls ein Unterterm $\bar{\sigma}\left\langle\bar{t}_{a}, \ldots, \bar{t}_{k-1}\right\rangle$ von $\sigma\left\langle t_{0}, \ldots, t_{n-1}\right\rangle$ ex.. so daB $\bar{\sigma}\left\langle\bar{t}_{a}, \ldots, \bar{t}_{k-1}\right\rangle$ und $\sigma^{\prime}\left\langle t_{0}^{\prime}, \ldots, t_{k-1}^{\prime}\right\rangle$ fastidentisch sind.

Ist $\bar{\sigma}\left\langle\bar{t}_{\square}, \ldots, \bar{t}_{k-1}\right\rangle$ unmittelbarer Unterterm van $\sigma\left\langle t_{a}, \ldots, t_{n-1}\right\rangle$, so bezeichnen wir $\sigma^{\prime}\left\langle t_{0}^{\prime}, \ldots, t_{k-1}^{\prime}\right\rangle$ als unmittelbaren Fastunterterm von $\sigma\left\langle t_{\square}, \ldots, t_{n-1}\right\rangle$. Es sei A eine endliche Menge mit $A \cap \Sigma=\emptyset$. Wir erweitern $\Sigma$ zu $\Sigma^{\prime}$ mit $\Sigma^{\prime}=A \cup \Sigma_{0}$

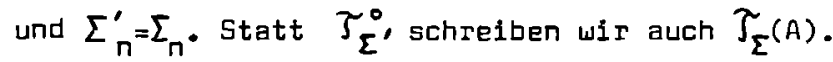


Definition: Sei $Q$ eine Menge sog. Zustände und $q, q^{\prime} \in$ Q. Dann heißt ein Paar $\left(q, \sigma\left\langle x_{0}, \ldots, x_{n}\right\rangle\right) \rightarrow\left(q^{\prime}, u\right)$ mit $\sigma \in \Sigma_{n}, u \in \mathcal{J}_{\Sigma}\left(\left\{x_{0}, \ldots, x_{n}\right\}\right), \pi \geqslant 0$, еiпe Eaumproduktion. Die $x_{i}$ bezeichnen wir als Variablen.

Definition: Ein 旦msystem $G$ über $\Sigma$ ist ein 4-tupel $(\Sigma, Q, 5, \pi)$ mit einer endlichen Menge $Q$ von Zuständen, einer endlichen Menge $\pi$ von Baumproduktionen über $Q$ und $\Sigma$, und 5 einer endlichen Untermenge van $Q \times \int_{\Sigma}^{0}$ (Startkanfigurationen).

Die wirkungsweise eines solchen Baumsystems ist gegeben durch:

Definition: Sei $(q, t),\left(q^{\prime}, t^{\prime}\right) \in Q \times \widetilde{\zeta}_{\Sigma}^{0} \cdot\left(q^{\prime}, t^{\prime}\right)$ wird aus $(q, t)$ direkt erzeugt $\left((q, t) \Rightarrow\left(q^{\prime}, t^{\prime}\right)\right)$, falls gilt:

(a) $t=\sigma\left\langle t_{0}, \ldots, t_{n-1}\right\rangle, n \geq 0$

(b) es gibt eine Baumproduktion $\left(q, G\left\langle x_{0}, \ldots, x_{n-1}\right\rangle\right) \rightarrow\left(q^{\prime}, u\right)$

(c) $t^{\prime}$ geht aus $u$ dadurch hervor, daß die $x_{i}$ in $u$ durch $t_{i}$ ersetzt шетden.

Mit $\stackrel{*}{\Longrightarrow}$ bezelchnen wir die reflexive transitive Hülle van $\Longrightarrow$.

4. Programme mit zweiparametrigen Prozeduren und Prozedurschachtelungstiefe $\leqslant 2$

Im folgenden beschäftigen wir uns mit Programmen, die nach der Umformung in ALGOL 60-P-G-Pragramme und der Beseitigung der Prazedurschachtelungen die allgemeine Gestalt

$$
\begin{aligned}
& \pi_{1}=\text { begin } \frac{\text { proc }}{\vdots} g_{1}\left(x_{1}^{1}, x_{1}^{2}\right) \text {; } \frac{\{\ldots\}}{\text { Hauptteil }} \text { von } g_{1} \\
& \text { proc } g_{n}\left(x_{n}^{1}, x_{n}^{2}\right) ;\{\ldots\} \text {; } \\
& \text { proc } f_{11}\left\langle\bar{x}_{1}^{1}, \bar{x}_{1}^{2}\right\rangle\left(y_{11}^{1}, y_{11}^{2}\right) \text {; } \\
& \underset{\vdots}{\operatorname{proc}} f_{1 m_{1}}\left\langle\bar{x}_{1}^{1}, \bar{x}_{1}^{2}\right\rangle\left(y_{1 m_{1}}^{1}, y_{1 m_{1}}^{2}\right) ;\{\ldots\} \text {; } \\
& \underset{\dot{\operatorname{proc}}}{i}{ }_{n 1}\left\langle\bar{x}_{n}^{1}, \bar{x}_{n}^{2}\right\rangle\left(y_{n 1}^{1}, y_{n 1}^{2}\right) ;\{\ldots\} \text {; }
\end{aligned}
$$

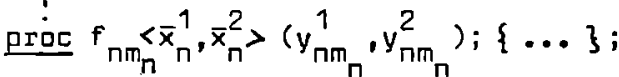

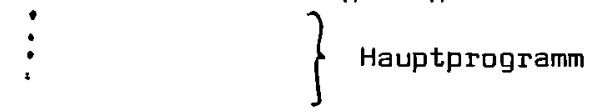

besitzen.

Im Hauptprogramm können nur Anweisungen der Art $g_{i}\left(g_{i^{\prime}}, g_{i^{\prime \prime}}\right), 1 \leqslant i, i^{\prime}, i^{\prime \prime} \leqslant n$ auf treten.

Bei den Anweisungen $a_{0}\left(a_{1}, a_{2}\right)$, die im Hauptteil von $g_{i_{1}}$ (i fest, $1 \leqslant i \leqslant n$ ) auftreten, kann $a_{k}, k=0,1,2$, ein formaler Identifikator $x_{i}$ bzw. $x_{i}^{2}$, ein globaler Identifikator $g_{1^{\prime}}\left(1 \leqslant i^{\prime} \leqslant n\right)$ oder ein Term $f_{i j}\left\langle x_{i}^{1}, x_{i}^{2}\right\rangle\left(1 \leqslant j \leqslant m_{i}\right)$ sein.

Bei den Anweisungen $a_{a}\left(a_{1}, a_{2}\right)$, die 1 m Hauptteil von $f_{i j}$ ( $i, j$ fest, $1 \leqslant i \leqslant n$, $1 \leqslant j \leqslant m_{i}$ ) auftreten, kann $a_{k}, k=0,1,2$, ein formaler Identifikator $y_{i j}^{1}$ oder $y_{i j}^{2}$ oder $\bar{x}_{i}^{1}$ oder $\bar{x}_{i}^{2}$, ein globaler Identifikator $g_{i^{\prime}}(1 \leqslant i \leqslant n)$ oder ein Term 
$f_{1 j^{\prime}}\left\langle\bar{x}_{i}^{1}, \bar{x}_{i}^{2}\right\rangle\left(1 \leqslant j^{\prime} \leqslant m_{i}\right)$ sein.

Die durch die Kopierregel entstehenden Terme im Hauptprogramm besitzen nun offensichtlich die Gestalt binärer Bäume, deren Blätter aus Identifikatoren $g_{i}$ bestehen. Den Zusammenhang zwischen den einzelnen Termen einer Anweisung zeigt

Satz 1: Bei allen Prozeduranweisungen $a_{0}\left(a_{1}, a_{2}\right)$, die in Hauptteilen von Programmen $\pi_{1}^{\prime}$ des Ausführungsbaumes $T_{\pi_{1}}$ des ALGOL 60-P-G-Programms $\pi_{1}$ auftreten gilt: Bei je zwei Untertermen $t_{l}, t_{k}$ van $a_{i}, a_{j}$ ist einer der $t_{l}, t_{k}$ Fastunterterm des andern.

Dies bedeutet insbesondere für die Terme $a_{i}, a_{j}$ d dab einer stets Fastunterterm des andern ist. Beschränken wir uns auf monadische Programme, so lassen sich die Aussagen über den Zusammenhang zwischen den einzelnen Termen weiter verschärfen :

Satz 2: Bei allen Prozeduranweisungen $a_{0}\left(a_{\eta}, a_{2}\right)$, die in Hauptteilen von Programmen $\widetilde{\pi}_{1}^{\prime}$ des Ausführungsbaumes $T_{\widetilde{\pi}_{1}}$ eines monadischen ALGOL 60-P-G-Pragramms $\widetilde{\pi}_{1}$ auftreten, gilt für je zwei Terme $a_{i}, a_{j}, 0 \leqslant i, j \leqslant 2$, eine der falgenden drei Eigenschaften:

a) $a_{i}=g_{i^{\prime}}$ ader $a_{j}=g_{i^{\prime}}, 1 \leqslant i^{\prime} \leqslant n, \quad$ oder

b) $a_{i}$ und $a_{j}$ sind fastidentisch oder

c) $a_{i}$ ist unmittelbarer Fastunterterm von $a_{j}$ ader umgekehrt.

Dieser 5atz zeigt, daß sich die einzelnen Terme nur in dem unmittelbaren Bereich um die wurzel unterscheiden. Durch das Notieren der Unterschiede zwischen den einzelnen Termen in einem Index an der Uurzel, lassen sich die einzelnen

Terme der Prozeduranweisungen $a_{0}\left(a_{1}, a_{2}\right)$ zu einem Term zusammenfassen. Samit läßt sich zu unserem Programm $\widetilde{\pi}_{1}$ ein Baumsystem $G_{\pi_{1}}$ konstruieren mit der.Eigenschaft

Satz 3: Eine Prozedur $p$ in $\widetilde{\pi}_{1}$ ist genau dann formal erreichbar, falls es in $G_{\pi_{1}}$ eine Startkonfiguration $s$ und es eine durch p bestimmte Kanfiguration $s_{p}$ gibt, so dal $s \stackrel{*}{\Longrightarrow} s_{p}$.

Da letzteres jedoch entschieden werden kann [8], erhält man unmittelbar

Satz4: Es ist entscheidbar, ob eine Prozedur in einem monadischen ALGOL 60-PProgramm mit höchstens zweiparametrigen Prozeduren und Prozedurschachtelungstiefe $<2$ formal erreichbar ist.

5. Programme mit einparametrigen Prozeduren und Prozedurschachtelungstiefe $\leqslant 3$ Im falgenden beschäftigen wiI uns mit Programmen, die nach der Umformung in ALGOL 60-P-G-Pragramme und der Beseitigung der Prozedurschachtelung die allgemeine Gestalt 


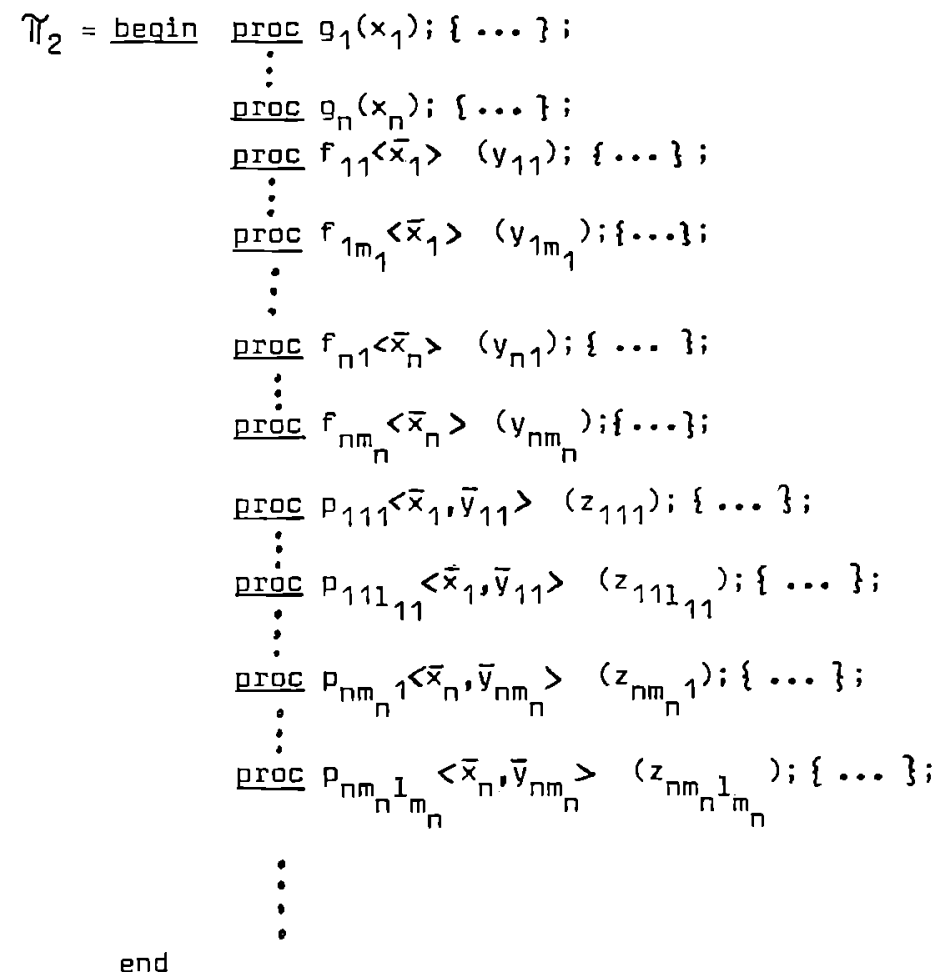

besitzen.

Bei einem solchen Programm kännen im Hauptprogramm nur Anweisungen der Art $g_{i}\left(g_{i}\right), 1 \leqslant i, i^{\prime} \leqslant n$, stehen.

Bei den Anueisungen $a_{0}\left(a_{1}\right)$, die im Hauptteil van $g_{i}$ (i fest, $1 \leqslant i \leqslant n$ ) auftreten, kann $a_{k}, k=0,1$, ein formaler Identifikator $x_{i}$, ein globaler Identifikator $g_{i}$ $\left(1 \leqslant i^{\prime} \leqslant n\right)$ oder ein Term $f_{i j}\left\langle x_{i}\right\rangle \operatorname{sein}\left(1 \leqslant j \leqslant m_{i}\right)$.

Bei den Anweisungen $a_{a}\left(a_{1}\right)$, die im Hauptteil von $f_{i j}(1, j$ fest, $1 \leqslant i \leqslant n$, $1 \leqslant j \leqslant m_{i}$ ) auftreten, kann $a_{k}, k=0,1$, ein formaler Identifikator alter Art $y_{i j}$, ein formaler Identifikator neuer Art $\bar{x}_{i}$, ein globaler Identifikator $g_{i}$ $\left(1 \leqslant i^{\prime} \leqslant n\right)$, ein Term $f_{i j^{\prime}}\left\langle\bar{x}_{i}\right\rangle \quad\left(1 \leqslant j^{\prime} \leqslant m_{i}\right)$ ader ein Term $p_{i j 1}\left\langle\bar{x}_{i}, y_{i j}\right\rangle \quad(1 \leqslant 1 \leqslant$ $\leqslant 1_{i j}$ ) sein.

Bei den Anweisungen $a_{0}\left(a_{1}\right)$, die im Hauptteil van $p_{i j 1}(i, j, 1$ fest, $1 \leqslant i \leqslant n$, $1 \leqslant j \leqslant m_{i}, 1 \leqslant 1 \leqslant 1_{i j}$ ) auftreten, kann $a_{k}, k=0,1$, ein formaler Identifikator alter Art $z_{i j l}$, ein formaler Identifikator newer Art $\bar{x}_{i}$ bzw. $\bar{y}_{i j}$, ein globaler Identifikator $g_{1^{\prime}}\left(1 \leqslant i^{\prime} \leqslant n\right)$, ein Term $f_{1 j^{\prime}}\left\langle\bar{x}_{1}\right\rangle \quad\left(1 \leqslant j^{\prime} \leqslant m_{1}\right)$ oder ein Term $p_{i j 1^{\prime}}\left\langle\bar{x}_{i}, \bar{y}_{i j}\right\rangle^{\prime}\left(1 \leqslant 1^{\prime} \leqslant l_{i j}\right)$ sein.

Beschränkt man sich nun auf monadische Programme, so läßt sich zeigen

Satz 5: Für alle Prozeduranweisungen $a_{0}\left(a_{1}\right)$, die in Programmen $\widetilde{\pi}_{2}$ des Ausführungsbaumes $T \widetilde{\pi}_{2}$ elnes monadischen ALGOL 60-P-G-Programms auftreten können, gelten die folgenden beiden Eigenschaften: 
Eigenschaft a:

1) $a_{0}=g_{i}$ oder $a_{1}=g_{i}, \quad 1 \leqslant i \leqslant n$, oder

2.) $a_{0}$ und $a_{1}$ sind fastidentisch oder

3) $a_{\square}$ besitzt nur einen einzigen unmittelbaren Unterterm und $\lambda$ ist linker unmittelbarer Unterterm von $a_{1}$ oder umgekehrt oder

4) $a_{a}$ besitzt einen unmittelbaren Unterterm $\lambda$ und $\lambda$ und $a_{1}$ sind fastidentisch oder umgekehrt oder

5) $a_{0}$ besitzt nur einen einzigen unmittelbaren Unterterm $\lambda$ und $\lambda$ ist linker unmittelbarer Unterterm eines unmittelbaren Unterterms von $a_{1}$ oder umgekehrt.

Eigenschaft $b$ :

Bei allen Termen $a_{i}, i=0,1$, der Art $a_{i}=\alpha\left\langle\beta_{1}, \beta_{2}\right\rangle$ gilt für die unmittelbaren Unterterme $\beta_{1}, \beta_{2}$ stets

1) $\beta_{2}=g_{i}, \quad 1 \leqslant i \leqslant n$, oder

2) $\beta_{1}$ und $\beta_{2}$ sind fastidentisch oder

3) $\beta_{2}$ besitzt nur einen einzigen unmittelbaren Unterterm $\lambda$ und $\lambda$ ist linker unmittelbarer Unterterm von $\beta_{1}$ oder

4) $\beta_{1}$ ist einziger oder linker unmittelbarer Unterterm vor $\beta_{2}$ oder

5) $\beta_{2}$ besitzt einen unmittelbaren Unterterm $\lambda$ und $\beta_{1}$ ist einziger oder linker unmittelbarer Unterterm von $\lambda$.

Dieser Satz zeigt, daß auch in diesem fall sich die einzelnen Terme nur in einem engen Bereich an der 5pitze unterscheiden und sich somit zu einem einzigen Term zusammenfassen lassen. Eigenschaft b) zeigt darüberhinaus, daß, falls ein Term zwei unmittelbare Unterterme besitzt, diese Unterterme sich ebenfalls nur unwesentlich unterscheiden. Dies ermöglicht uns die Codierung eines Terms in eine lineare Zeichenkette. Samit läßt sich in diesem Fall die Entscheidbarkeit der formalen Erreichbarkeit einer Prozedur über Stacksysteme zeigen.

Definition: Ein Stack-System ist ein 4-tupel $\gamma=(\pi, 4,5, \pi)$ mit

(i) Mist eine endliche nicht leere Menge (nicht terminale Symbole),

(ii) 4ist eine endliche nicht leere Menge (terminale Symbole) mit $\pi \cap 4=\pi$,

(iii) $5 \subset \& \gamma$ (Startworte),

(iv) $\pi$ ist eine endliche Menge von Produktionsregeln der Formen

(mit $5^{\prime}, S^{\prime \prime} \in M ; A, \theta \in \& ; Q_{1} Q_{1}, Q_{2}$ Variablen über $4^{*}$ ):

1) $\mathrm{QAS}^{\prime} \rightarrow$ QABS"

2) $\mathrm{QAS}^{\prime} \longrightarrow \mathrm{QAS}^{\prime \prime}$

3) $\mathrm{QAS}^{\prime} \rightarrow \mathrm{QS}$ "

4) $Q_{1} A S^{\prime} Q_{2} \rightarrow Q_{1} A S^{\prime \prime} Q_{2}$

5) $\mathrm{Q}_{1} A \mathrm{SL}_{2} \rightarrow \mathrm{Q}_{1} \mathrm{~S}^{\prime \prime} \mathrm{AQ_{2 }}$ 
6) $\mathrm{Q}_{1} \mathrm{AS}^{\prime} \mathrm{Q}_{2} \rightarrow \mathrm{Q}_{1}{ }^{A B S} \mathrm{~S}_{2}$

Die Arbeitsweise eines Stack-Systems ist gegeben durch

Definition: $x \Rightarrow y$ mit $x, y \in d^{*} \eta 4^{*}$

*: falls $x=w y, y=$ uy $y^{\prime}$ mit $w \in \mathcal{f}^{*}, y, y^{\prime} \in 4 \gamma$ ist und in $\pi$ eine Produktion $Q y \rightarrow Q y^{\prime}$ ex. oder falls $x=w y w^{\prime}, y=w y^{\prime} w^{\prime}$ mit

$w^{\prime} w^{\prime} \in \mathcal{f}^{*}, y, y^{\prime} \epsilon 4 \pi 4$ ist und in $\pi$ eine Produktion $Q_{1} y Q_{2} \rightarrow Q_{1} y Q_{2}$ ex..

Mit $\stackrel{\gtrless}{\Longrightarrow}$ bezeichnen wir die transitive reflexive Hülle von $\Longrightarrow$ •

Es läßt sich пun zеigen:

Satz 6: $Z u \widetilde{\pi}_{2}$ läßt sich ein Stack-System $\gamma_{\pi_{2}}$ konstruieren mit der Eigenschaft: Eine Prazedur $q$ in $\widetilde{\pi}_{2}$ ist genau dann farmal erreichbar, falls es in $\gamma_{\pi_{2}}$ ein Startwort $s$ und ein durch q begtimmtes wort tw $\in 4^{*} \gamma$ gibt, so daß gilt $\quad \stackrel{*}{\Rightarrow} t w$.

Da letzteres jedoch entscheidbar ist [1], erhält man

Satz 7: Es ist entscheidbar, ob eine Prozedur in einem monadischen ALGDL 60P-Programm mit höchstens einparametrigen Prozeduren und Prozedurschachtelungstiefe $\leqslant 3$ farmal erreichbar ist.

\section{Schlußbemerkungen}

Für monadische Programme haben wir also folgendes Diagramm

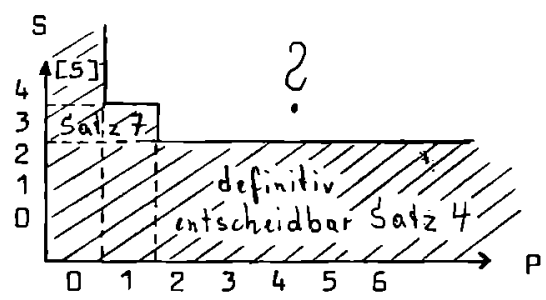

dessen definitiver Entscheidbarkeitsbereich über den früheren hinausragt.

Bei höherer Schachtelungstiefe als 3 im einparametrigen monadischen fall wird die formale Erreichbarkeit von Prozeduren wahl auch noch entscheidbar sein. Bei höherer Parameterzahl und Schachtelungstiefe $\geqslant 3$ wagen. wir im Moment noch keine Prophezeiung. 
Literatur:

[1] Ginsburg,5.,Greibach,S.A. und Harrisan,M.A.:

Stack automata and compiling, J.ACM. 14,1 (Jan.67), 172-201

[2] Brainerd,W.: Tree generating regular systems, Information and Control 14 (1969), 217-231

[3] Doner,J.: Tree acceptors and some of their applications, Journal of Computer and 5ystem Sciences 4 (1979), 406-451

[4] Kaufholz,G.,Lippe,W.M.: A note on a paper of H. Langmaack about procedure parameter transmission, Bericht $A$ 74/06 des Fachbereichs Angewandte Mathematik und Informatik der Universität des Sariandes (1974)

[5] Langmaack,H.: Dn correct procedure parameter transmission in higher programming languages, Acta Informatoca Vol.2,(1973)

[G] Langmaack, H.: Dn procedures as apen subroutines I, II Acta Informatica Vol.2, 1973 und Val.3,(1974)

[7] Lippe,w.M.: Entscheidbarkeitsprobleme bei der Übersetzung von Programmen mit einparametrigen Prozeduren, Lecture Notes in Camputer Scince Nr.7, Springer Verlag (1974)

[8] Rounds,w.C.: Mappings and grammars on trees, Mathematical systems theorie, Vol. 4, Nr.3, 257-287 